\title{
Improving management of severe sepsis and uptake of sepsis resuscitation bundle in an acute setting
}

\author{
Sumitra Kafle, Navdeep Nath
}

Worcestershire Royal Hospital

\begin{abstract}
Severe sepsis still remains a major cause of morbidity and mortality, claiming between 36,000 to 64,000 lives annually in the UK, with a mortality rate of $35 \% .[1,2]$
\end{abstract}

The project aims to measure the management of severely septic patients in acute medical unit (AMU) in a district general hospital against best practice guidelines, before and after a set of interventions aiming to optimise patient management and outcomes.

All new admissions who met the criteria for sepsis in AMU over a two week period were evaluated. Those who met the criteria for severe sepsis were further analysed. The criteria evaluated were time to first administration of oxygen, intravenous fluids, antibiotics, the taking of blood cultures, other relevant bloods tests (including lactate) and urine output monitoring. A re-audit was completed after the introduction of a set of interventions which included a "sepsis box."

A total of 32 patients (19 Males, 13 Females) were identified in the pre-intervention group. Twenty-two of these patients met the criteria for severe sepsis. Only 15 out of $32(47 \%)$ had their lactate measured. Ten out of $22(45 \%)$ received fluids within an hour. Twelve out of $22(55 \%)$ had their blood culture sample taken after administration of antibiotics and only 12 out of $22(55 \%)$ had antibiotics administrated within an hour of medical assessment.

Post-intervention the results however improved dramatically. A total of 30 patients were identified in the post-intervention group (12 Males, 18 Females). Antibiotics administration within an hour went up by $22 \%$. Lactate was performed in $26 / 30(87 \%)$ patients presented with sepsis compared to $47 \%$ in the pre-intervention group. Similarly, identification of severe sepsis, and administration of intravenous fluids also showed improvement ultimately improving patient safety.

Following the initial success, the trial was repeated over three months period, which showed sustainable improvement.

\section{Problem}

During the post-take ward rounds, it was evident that there was a significant delay in the investigations and treatment of new patients presenting with sepsis in an acute medical unit (AMU). The delay may result in worsening sepsis and increase morbidity and mortality. There were no clear trust guidelines on management of sepsis and many of the nurses working in AMU were recently qualified and did not have prior knowledge of sepsis and severe sepsis. The international sepsis guidelines were not being implemented correctly due to several factors.

One of the problems was a busy AMU where tools required to complete the sepsis resuscitation bundle were kept in different parts of the AMU. As a result of this, patients did not have a full set of relevant blood tests (including lactate) performed to determine the severity of sepsis. For those with established severe sepsis, blood cultures were not always taken and antibiotics as well as intravenous fluids were not administrated within an hour of medical assessment. Frequently, blood cultures were taken several hours after the administration of antibiotics.

\section{Background}

Sepsis is a common condition with a major impact on healthcare resources and expenditure.[1] It accounts for mortality rate of $35 \%$; 20,000 deaths per day worldwide and 64,000 deaths annually in the UK.[1] There are few disease processes with such a high mortality. An admission with severe sepsis places the patient at six to ten fold higher risk of mortality than if they were admitted with an acute myocardial infarction and four to five times greater risk than if they had suffered an acute stroke.[1]

Therefore it is important that sepsis is recognised and managed early. Research has shown that early goal directed therapy and resuscitation bundle improves survival rates in sepsis. [1,2,3,4] One such resuscitation bundle is sepsis six which is deliverable in the general ward setting. $[1,2,4]$ The sepsis six comprises of three diagnostic/monitoring steps and three therapeutic interventions.[2,4]

Theses are:

1. Deliver high-flow oxygen

2. Take blood cultures and other cultures, consider source 
control

3. Administer empirical intravenous (IV) antibiotics

4. Measure serum lactate or alternative

5. Start IV fluid resuscitation using Hartmann's or equivalent

6. Commence accurate urine output measurement.

\section{Baseline measurement}

All new admissions over a two week period who met the criteria for sepsis in AMU were included in the audit. Then the patient's notes were evaluated to identify if they were septic and/or severely septic. The following criteria was used to identify patients with sepsis:

Clinical evidence of infection or Patient at risk score (PARS) of $\geq 3$ and any two of the following present?

1. Temperature less than 36 ? $\mathrm{C}$ or more than 38 ?C

2. Respiratory rate more than $20 / \mathrm{min}$

3. Acutely altered mental state

4. HR more than 90

The following criteria was used to identify patients with severe sepsis:

1. Systolic BP less than 90

2. MAP less than 65

3. Lactate greater than two

4. Other evidence of organ dysfunction: (Creat $>177$, Bili $>$ 34 , Plt $<100$, INR $>1.5$, urine output $<0.5 \mathrm{~mL} / \mathrm{kg} / \mathrm{hr}, \mathrm{SpO} 2<$ $90 \%)$.

Those who met the criteria for severe sepsis were analysed to identify the following:

- Time to first administration of oxygen, intravenous fluids and antibiotics

- Taking of blood cultures, other relevant bloods tests (including lactate) and urine output monitoring.

A total of 32 patients (19 Males, 13 Females) were identified in the pre-intervention group. Twenty-two of these patients met the criteria for severe sepsis. Only 15 out of $32(47 \%)$ had their lactate measured. Ten out of $22(45 \%)$ received fluids within an hour. Only 12 out of 22 (55\%) had their blood culture taken and the same percentage had their antibiotics administrated within an hour of medical assessment. Of those who had blood cultures taken, 8/12 $(67 \%)$ were taken after the administration of antibiotics.

See supplementary file: ds4186.docx - "Pre-intervention results and Sepsis six pathway"

\section{Design}

It was obvious that multifactorial approach was necessary to improve management of sepsis. There were two important factors highlighted during the audit. One was lack of understanding of sepsis and management of sepsis among healthcare professionals involved in the care of newly admitted septic patients.

Second was the time taken to collect all the necessary equipment required to complete the sepsis six bundle in a busy and disorganised AMU. Many highlighted the fact that culture bottles were not always available in AMU and had to be delivered by a porter once ordered from the labs which wasted hours. To target the above problems, following solutions were implemented:

1. Sepsis boxes with all the necessary equipment to enable medical staff to implement all the facets of the sepsis guideline within an hour of medical assessment

2. Teaching sessions on sepsis for nurses working in AMU

3. Sepsis posters to display in AMU

4. Easy to follow sepsis guidelines/pathway

5. Inform the doctors with a clerking role about the sepsis pathway.

In order to make the program sustainable, sepsis protocol would have to be incorporated in hospital guidelines. These guidelines should be available in the hospital intranet for ease of access. Along with this, A\&E as well as AMU staff should have continuing training and education on sepsis. The aim of the audit was to introduce such guidelines and create a platform for sustainable improvement.

\section{Strategy}

PDSA cycle 1: A sepsis six pathway was created as this was not already available in the trust. Meetings were held to design the pathway in such a way that it stood out from the other treatment pathways available in the hospital. We decided to make it as simple as possible and use blue background with black writing. This received very good feedback as it was not yet another piece of form to be filled in by nurses and doctors.

PDSA cycle 2: To raise awareness of sepsis, we designed posters which were displayed in AMU. The posters stood out due to red background and again had minimal writing. Along with this, teaching sessions were held for nurses working in AMU. The teaching focused on recognition of sepsis and nurses' role in management of sepsis. This was received very well.

PDSA cycle 3: Due to lack of space in the clinical rooms, a meeting was held with AMU managers to help identify a potential space where sepsis boxes could be kept so that they were readily available but without taking too much space. As a result of this, the size of the boxes had to be reduced so catheters were not included in the boxes. However, a reminder of the sepsis six pathway was stuck to the top of the box. Six sepsis boxes were available in each clinical room.

PDSA cycle 4: The sepsis pathway as well as summary of the audit was emailed to all the doctors with a clerking role to encourage and facilitate an improvement in management of sepsis. Questionnaire filled out by doctors revealed that sepsis box was found to be a very useful tool to facilitate management of sepsis and save time. 
PDSA cycle 5: After three weeks of interventions, we conducted a re-audit over a two weeks period. The results were encouraging. Following this, the next few months were spent incorporating sepsis protocol in the hospital policies and guidelines. This included several meetings with microbiologists, infection disease consultant, AMU, and A\&E department. The sepsis pathway was edited several times. Eventually a decision was made to make it simple just like it was initially designed. However, the A\&E department decided to make their own sepsis pathway which was more comprehensive. These guidelines were then available on the intranet.

PDSA cycle 6: Four nurses were recruited as sepsis nurses in AMU who played a vital role in day to day up-keeping of sepsis boxes as well as training and educating their fellow colleagues.

PDSA cycle 7: After several months, a re-audit was conducted over a three months period - 10 patients with sepsis per week. Following the overall success of the project, the results were presented in a board meeting. This led to a decision for guidelines to be implemented across both acute trusts.

\section{Results}

Results were initially collected three weeks post-intervention, which showed dramatic improvement. A total of 30 patients were identified in the this post-intervention group (12 Males, 18 Females). There were 19/30 patients who qualified as severely septic. Antibiotics administration within an hour went up by $22 \%$ and lactate was performed in $26 / 30$ (87\%) patients presenting with sepsis compared to $47 \%$ in the pre-intervention group. Although the taking of blood cultures within an hour did not show a big improvement (11/19), it was interesting to see that majority of them were taken before the administration of the antibiotics (8/11) and not after. Similarly, identification of severe sepsis, and administration of intravenous fluids also showed improvement ultimately improving patient care.

The re-audit conducted over three months period, starting from April 2014 also showed sustainable improvement. Total of 10 patients per week were included in the audit. The results are displayed in the run charts attached. To make the results as comparable as possible, the pre-intervention and three weeks post-intervention data have been dissected so that each data point represents 10 patient giving a total of 18 data points.

The prescribing of oxygen does show a random variation, suggesting that the intervention did not have any effect on the results, although all the other measurements do show a nonrandom variation. Blood cultures taken within an hour and blood cultures taken before the administration of first dose of antibiotics show a shift pattern. Similarly, measurement of lactate, IVI within an hour and antibiotics within an hour show a run pattern.

The results show a negative trend for weeks one to three post intervention. Although the reason for this is not clear, it could be speculated that this was because data was collected at the start of April and hence it coincided with the change of doctors' placement. New doctors working in AMU and A\&E were perhaps not familiar with the sepsis guidelines or the availability of sepsis boxes.
However, it was encouraging to see an upward trend after the first few weeks.

See supplementary file: ds4112.docx - "Pre and post intervention results"

\section{Lessons and limitations}

I have learned that even the smallest change or improvement can still have a significant impact on clinical practice. This audit has highlighted the importance of evidence based medicine in making policies and how it shapes our current practice. After just three weeks it was evident that education, clinical pathway, and sepsis box together made a positive difference in management of sepsis. Given time and continued training, there is no reason why we cannot achieve $100 \%$ in all the aspects of sepsis six management.

Limitations of this project would be the fact that we have not measured if the improvement in management of sepsis has subsequently improved the length of stay in hospital or the rate of mortality. However, given the research we anticipate a positive outcome.

Following this study, all the junior doctors will now receive a lecture on management of sepsis at the start of their year and the trust plans to roll out sepsis boxes throughout the hospital. An infectious disease consultant has taken the role of championing management of sepsis in the hospital so that there is continuity of care.

\section{Conclusion}

Before the intervention, management of severely septic patients in our hospital fell short of best practice standards. However, this improved greatly with the uptake of education program and sepsis box, helping to identify severely septic patients earlier and therefore to be able to start appropriate interventions earlier. This subsequently improved patient care.

\section{References}

1. Daniels, R. Surviving the ?rst hours in sepsis: getting the basics right (an intensivist's perspective). J Antimicrob Chemother 2011;(66): ii11-ii23.

2. Robson W, Nutbeam T, Daniels R. Sepsis- a need for prehospital intervention? EMJ 2009;26(7):535-8.

3. Dellinger RP, Levy MM, Rhodes A et.al. Surviving sepsis campaign: international guidelines for management of severe sepsis and septic shock: 2012. Crit Care Med 2013;41(2):580-637.

4. Daniels R, Nutbeam T, McNamara G, Galvin C.The sepsis six and the severe sepsis resuscitation bundle: a prospective observational cohort study. EMJ 2011;28(6): 507-12.

\section{Declaration of interests}


Nothing to declare.

\section{Acknowledgements}

Dr Charlotte Braniff, Dr Jane Stockley, Dr M Ling, Dr R. Alexander. 